\title{
ATRIBUTOS FÍSICOS INDICADORES DA QUALIDADE DO SOLO SOB SISTEMAS DE MANEJO NA BACIA DO ALTO DO RIO GRANDE-MG ${ }^{1}$
}

\author{
Physical properties as indicators of soil quality on different management systems at the alto Rio \\ Grande, state of Minas Gerais, Brazil
}

Rubens Ribeiro da Silva², Marx Leandro Naves Silva ${ }^{3}$, Mozart Martins Ferreira ${ }^{3}$

\begin{abstract}
RESUMO
O uso agrícola provoca alteração nos atributos físicos do solo. Normalmente essa alteração induz uma deterioração de sua qualidade, em decorrência da retirada da cobertura vegetal e o excessivo uso da mecanização. Diante disso, avaliou-se as alterações de alguns atributos indicadores da qualidade física de um Latossolo Vermelho-Amarelo Ácrico típico, sob diferentes sistemas de manejo em relação ao cerrado nativo. Para tanto foram coletadas amostras de solo nas camadas de 0-10, 10-20 e 20-30 cm na região Campos das Vertentes, na Bacia Alto do Rio Grande, MG. Os atributos físicos avaliados foram: estabilidade de agregados, expresso pelo diâmetro médio geométrico; densidade do solo; porosidade do solo e resistência do solo à penetração. Os sistemas de manejo avaliados foram: cultivo convencional com batata (CCB); cultivo convencional com batata sucedido por aveia em rotação com milho (CCBAM); cultivo convencional com milho (CCM); plantio direto com milho (PDM); cultivo com eucalipto (CE) e cerrado nativo (CN) como testemunha. A densidade do solo aumentou nos sistemas CCBAM e PDM, em relação ao cerrado nativo. O volume total de poros foi reduzido nos sistemas CCB, CCBAM e PDM, mesmo com o pouco tempo de cultivo nos sistemas CCB e CCBAM. Os sistemas convencionais CCB, CCBAM e CCM não alteraram a estabilidade de agregado do solo o suficiente para diferir do cerrado nativo. Os sistemas com menor revolvimento estão estabelecendo uma nova condição de equilíbrio. O menor revolvimento do solo, o acúmulo de matéria orgânica e a ação agregante do sistema radicular das gramíneas nos sistemas PDM e CE foram benéficos na manutenção da qualidade do solo. A resistência à penetração foi reduzida nas camadas superficiais nos sistemas convencionais CCB, CCBAM e CCM em função da mobilização do solo. Não houve diferenças significativas na permeabilidade do solo à água entre os sistemas de manejo (CCB, CCBAM, CCM e PDM) estando os valores na faixa de 35,4 a 54,6 $\mathrm{mmh}^{-1}$, sendo a mesma classificada como lenta a moderada; estes valores foram bem inferiores aos do sistema em equilíbrio $(\mathrm{CN})$.
\end{abstract}

Termos para indexação: Cerrado, Latossolo, plantio direto, batata, milho.

\begin{abstract}
The agriculture use causes alterations on the soil physical attributes. That a alterations usually indices a deterioration of its quality, due to removal of the canopy and excessive use of the mechanization. This work aimed to evaluate the alterations of soil physical properties as indicators of soil quality of a typic Acric Red-Yellow Latosol (Oxisol), under different management systems, compared to the native vegetation. Soil samples were collected at the 0-10,10-20, and 20-30-cm depths in the region of Campos das Vertentes, at the Alto do Rio Grande, MG. The evaluated attributes were: aggregate stability in water, expressed for the geometric mean diameter, bulk density, and porosity. The following management systems were evaluated: conventional tillage with potato (CTP); conventional tillage with potato, followed by oat in rotation with corn (CTPOC); conventional tillage with corn (CTC); notill with corn (NTC); eucalyptus forest (EF); and native vegetation (NV). The bulk density increased in the systems CTPOC and NTC, compared to the native vegetation. The total volume of pores was reduced in the systems CTP, CTPOC, and NTC, even within the short growing time of the systems CTP and CTPOC. The conventional systems CTP, CTPOC and CTC did not change the soil aggregate stabilty enough to differ of the native vegetation. The systems with less canopy removal are establishing a new equilibrium condition. The slighter soil tillage, the higher organic matter content, and the grass root aggregating action in the systems NTC and EF were benefic for the soil quality. The penetration resistance was reduced in the superficial layers in the conventional systems, CTP, CTPOC, and CTC, due to the soil till. There were no significant differences in permeability of soil to water among the management systems (CTP, CTPOC, CTC and NTC), with the values ranging from 35,4 to $54,6 \mathrm{mmh}^{-1}$ being classified as slow to moderate; these values were rather inferior than the equilibrium system $(\mathrm{NV})$.
\end{abstract}

Index terms: Cerrado, Oxisol, Latosol, No-till, Potato, maize.

(Recebido para publicação em 28 de julho de 2003 e aprovado em $1^{\circ}$ de abril de 2005)

\section{INTRODUÇÃO}

Os solos sob cerrado no Brasil, em geral, apresentam condições físicas favoráveis para a agricultura, e vêm sendo gradativamente explorados com culturas anuais, pastagens e mais recentemente com reflorestamentos. No entanto, quando submetidos a determinados sistemas de manejo, tendem a um novo estado de equilíbrio, refletido em diferentes manifestações de seus atributos físicos, os quais poderão ser desfavoráveis à conservação do solo e a produção das culturas.

\footnotetext{
${ }^{1}$ Parte da dissertação apresentada à Universidade Federal de Lavras/UFLA, pelo primeiro autor, para obtenção do título de Mestre em Solos Nutrição de Plantas. 2 Professor da Universidade Federal do Tocantins/UFT - Caixa Postal 66 - 774.000-000 - Gurupi, TO - rrs2002@uft.edu.br

3 Professores do Departamento de Ciência do Solo - Universidade Federal de Lavras/UFLA - Bolsista do CNPq - Caixa Postal 3037 - $37.200-000$ Lavras, MG-marx@ufla.br -mozartmf@ufla.br.
} 
Segundo Vieira \& Muzille (1995), os efeitos diferenciados sobre os atributos físicos devido ao tipo de preparo de solo adotado em cada sistema de manejo, são dependentes da intensidade de revolvimento, do trânsito de máquinas, do tipo de equipamento utilizado, do manejo dos resíduos vegetais e das condições de umidade do solo no momento do preparo. O manejo incorreto de máquinas e equipamentos agrícolas, leva à formação de camadas subsuperficiais compactadas. Esse manejo tem sido apontado por Campos et al. (1995), como uma das principais causas de degradação da estrutura do solo e da redução da produtividade das culturas.

A relação entre o manejo e a qualidade do solo pode ser avaliada pelo comportamento das propriedades físicas, químicas e biológicas do solo (DORAN \& PARKIN, 1994). O efeito do manejo sobre as propriedades físicas do solo é dependente da sua textura e mineralogia, as quais influenciam a resistência e a resiliência do solo a determinada prática agrícola (SEYBOLD et al., 1999).

Dentre as propriedades físicas do solo, a estrutura é uma propriedade sensível ao manejo e pode ser analisada segundo variáveis relacionadas a sua forma (ALBUQUERQUE et al., 1995) e ou a sua estabilidade (CAMPOS et al., 1995). De modo geral, com o aumento da intensidade de cultivo tem sido observada alteração no tamanho dos agregados do solo, aumento da densidade do solo, redução da porosidade total e aumento da resistência do solo à penetração (ALBUQUERQUE et al., 1995; ALVARENGA \& DAVIDE, 1999; ANJOS et al., 1994; D'ANDRÉA, 2001; SILVA \& MIELNICZUK, 1997).

Estudos relacionando atributos físicos do solo e aspectos de qualidade e produtividade de tubérculos de batata inglesa em Latossolos e Cambissolos foram desenvolvidos por Rachwal \& Dedecek (1996), segundo os autores em condições de solo e clima desfavoráveis, com o processo erosivo estabelecido, porosidade efetiva inferior a $0,1 \mathrm{~m}^{3} \mathrm{~m}^{-3} \mathrm{e}$ déficit hídrico, contribuíram para reduzir a produção comercial e aumentar a quantidade de tubérculos rachados, notadamente no Cambissolo severamente erodido.

Assim, o monitoramento da qualidade do solo é necessário e pode ser realizado mediante avaliação dos seus atributos físicos, que são importantes para a sustentabilidade dos sistemas agrícolas. No monitoramento da qualidade do solo, os atributos usados como indicadores de mudanças devem ser sensíveis ao manejo numa escala de tempo que permita a verificação (DORAN \& PARKIN, 1994).
Diante disso, avaliou-se a alteração em atributos indicadores da qualidade física de um Latossolo VermelhoAmarelo Ácrico típico, sob diferentes sistemas de manejo em relação ao cerrado nativo.

\section{MATERIALEMÉTODOS}

O trabalho foi realizado no município de São João Del Rei na bacia do Alto Rio Grande, Estado de Minas Gerais, em clima Cwa (clima temperado suave mesotérmico), segundo Köppen, com temperatura média de $18^{\circ} \mathrm{C}$ no mês mais frio e a média de $22^{\circ} \mathrm{C}$ no mês mais quente, sendo um verão chuvoso e inverno seco. A precipitação média anual é de $1.435 \mathrm{~mm}$, concentradas entre os meses de novembro a abril. O solo estudado foi um Latossolo Vermelho-Amarelo Ácrico típico de textura muito argilosa (66\% argila; $22 \%$ silte e $12 \%$ areia), (EMBRAPA, 1999). Esse solo foi desenvolvido sobre um substrato geológico de rochas metapelíticas pobres do grupo São João Del Rei (filito), (BRASIL, 1983). A caracterização mineralógica foi realizada por Chagas et al. (1997), quando foram verificados os valores $\left(\mathrm{g} \mathrm{kg}^{-1}\right)$ de: $\mathrm{SiO}_{2}: 161$ e 158; $\mathrm{Al}_{2} \mathrm{O}_{3}: 260$ e $291 ; \mathrm{Fe}_{2} \mathrm{O}_{3}: 145$ e $157 ; \mathrm{TiO}_{2}: 10,8$ e 12,$8 ;$ ki: 1,05 e 0,92 e kr: 0,78 e 0,69, nas profundidades de 0-26 e 69-106 $\mathrm{cm}$ respectivamente, (ki: relação molecular sílica/alumina; kr: relação molecular sílica/alumina mais óxido de ferro).

$\mathrm{O}$ delineamento experimental utilizado foi o de blocos inteiramente casualizados, com três repetições e 15 tratamentos, além de uma testemunha adicional (cerrado nativo). Os tratamentos foram dispostos em um esquema fatorial $5 \times 3$, representado por cinco sistemas de manejo e três profundidades de amostragem (0-10, 10-20 e 20-30 cm). Os sistemas estudados foram: CCB: $\left(21^{\circ} 42^{\prime} \mathrm{S}\right.$ e $\left.44^{\circ} 21^{\prime} \mathrm{W}\right)$ Cultivo convencional com batata, safra 00/01, amostrado após a colheita ( 1 ano de uso); CCBAM: $\left(21^{\circ} 41^{\prime}\right.$ 'S e $4^{\circ} 39^{\prime} \mathrm{W}$ ) Cultivo convencional com batata, sucedido com aveia, e rotacionado com milho na safra 00/01, após uma subsolagem pós-colheita da batata na safra 99/00, amostrado 15 dias após o plantio direto do milho ( 2 anos

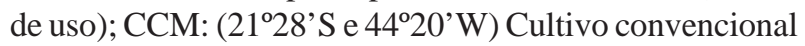
com milho na safra 00/01, em rotação com feijão da safra 99/00, amostrado aos 29 dias após o plantio (2 anos de uso); PDM: (213' $\mathrm{S}$ e $\left.44^{\circ} 18^{\prime} \mathrm{W}\right)$ Plantio direto com milho no ano agrícola 96/01, após o cultivo convencional com batata na safra $86 / 87$, arroz nas safras $87 / 89$ e milho convencional de 88/96, amostrado aos 52 dias após o plantio (15 anos de uso, sendo 5 anos sob PDM com o cultivo da gramínea (Brachiaria brizanta) em sobressemeadura durante 2 anos nas entrelinhas da cultura

Ciênc. agrotec., Lavras, v. 29, n. 4, p. 719-730, jul./ago., 2005 


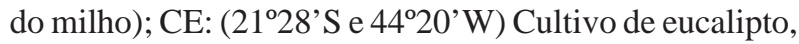
implantado no ano agrícola 78/79, sem aplicação de práticas

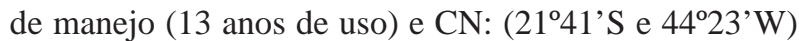
Cerrado nativo, constituído de vegetação primária remanescente, representada pelo cerrado tropical subcaducifólio. Na seleção dos sistemas de manejo estudados buscou-se uma melhor representação do uso agrícola na região e a proximidade dos sistemas de manejo sobre a mesma classe de solo, possibilitando maior controle do erro experimental, por meio de observações da posição topográfica na topossequência e da homogeneidade do perfil do solo nos locais de coleta de solo.

A parcela experimental foi constituída por uma mini trincheira ( 40 x 40 x $40 \mathrm{~cm}$ ), aberta nas entre-linhas de cada sistema de manejo selecionado. A amostragem do solo de cada parcela experimental foi realizada em janeiro 2001.

$\mathrm{Na}$ caracterização química do solo estudado foi determinado o teor de carbono orgânico total (Quadro 1), por meio de oxidação a quente com dicromato de potássio e titulação com sulfato ferroso amoniacal.

A estabilidade dos agregados foi determinada utilizando-se o peneiramento em água, após um préumedecimento lento por capilaridade, sobre papel de filtro umedecido (EMBRAPA, 1997). Para a separação das classes de tamanho dos agregados foram utilizadas peneiras com malhas: $2 \mathrm{~mm} ; 1 \mathrm{~mm} ; 0,5 \mathrm{~mm} ; 0,25 \mathrm{~mm}$ e 0,105 $\mathrm{mm}$. O diâmetro médio geométrico (DMG) foi calculado usando a expressão:

\section{$D M G=\exp \left[O ́\left(w_{i^{*}} \operatorname{Ln} x_{m}\right) / O ́ w_{i}\right]$.}

em que:

DMG: diâmetro médio geométrico (mm);

$\mathrm{w}_{\mathrm{i}}$ : massa dos agregados de cada classe de tamanho $(\mathrm{g})$;

Ln $x_{m}$ : logaritmo natural do diâmetro médio de cada classe de tamanho;

Ów $w_{\mathrm{i}}$ : massa total da amostra (g).

A concentração dos agregados maiores que $2 \mathrm{~mm}$ foi calculada pela expressão:

$$
\%>2=100{ }_{*}\left[\left(\mathrm{w}_{>2}\right) / \mathrm{O}_{\mathrm{i}}\right]
$$

em que:

$\%>2$ : porcentagem de agregados maiores que $2 \mathrm{~mm}$; w > 2: massa dos agregados de cada classe de tamanho $(\mathrm{g})$.

Para a determinação da densidade do solo pelo método do anel volumétrico (BLAKE \& HARTGE, 1986), foram coletadas amostras indeformadas com amostrador de Uhland em cilindros com volume médio de $313,9 \mathrm{~cm}^{3}$. Na determinação da porosidade total do solo foi seguida a metodologia definida por Danielson \& Sutherland (1986). A distribuição de poros por tamanho foi determinada utilizando um funil com placa porosa, na unidade de sucção a $60 \mathrm{~cm}$ de altura de coluna d'água, onde ocorreu a sucção da água contida na macroporosidade das amostras

QUADRO 1 - Teores de carbono orgânico total em um Latossolo Vermelho-Amarelo Ácrico típico sob diferentes sistemas de manejo em relação ao cerrado nativo.

\begin{tabular}{|c|c|c|c|c|c|c|c|}
\hline \multirow{2}{*}{ Profundidade $(\mathrm{cm})$} & \multicolumn{6}{|c|}{ Sistema de manejo ${ }^{(1)}$} & \multirow{2}{*}{ Média } \\
\hline & CCB & ССВАМ & CCM & PDM & $\mathbf{C E}$ & $\mathbf{C N}$ & \\
\hline & \multicolumn{6}{|c|}{ - } & \\
\hline $0-10$ & 24,36 & 25,71 & 28,42 & 28,81 & 24,0 & 32,87 & $27,36 a$ \\
\hline $10-20$ & 20,49 & 19,33 & 22,81 & 28,42 & 22,43 & 21,46 & $22,49 b$ \\
\hline $20-30$ & 16,05 & 16,82 & 16,05 & 21,46 & 17,21 & 16,82 & $17,40 \mathrm{c}$ \\
\hline Média & $20,30 \mathrm{~B}$ & $20,62 \mathrm{~B}$ & $22,43 \mathrm{~B}$ & $26,23 \mathrm{~A}$ & $21,20 \mathrm{~B}$ & $23,71 \mathrm{~A}$ & \\
\hline C.V. Sistema & $(\%)$ & 15,57 & & & & & \\
\hline C.V. Total & $(\%)$ & 12,58 & & & & & \\
\hline
\end{tabular}

(1) CCB (Cultivo convencional de batata); CCBAM (Cultivo convencional de batata, sucedido por aveia após uma subsolagem, e rotação com milho); CCM: (Cultivo convencional com milho em rotação com feijão); PDM (Plantio direto com milho, após os cultivos convencionais com batata, arroz e milho); CE (Cultivo de eucalipto) e CN (Cerrado nativo). Médias seguidas pela mesma letra, minúscula na coluna e maiúscula na linha, não diferem estatisticamente entre si, pelo teste de Scott e Knott ao nível de $5 \%$ de probabilidade. 
previamente saturadas por 48 horas. O volume de água retido no solo após o equilíbrio foi considerado correspondente à microporosidade. A resistência à penetração vertical foi determinada utilizando o penetrômetro de impacto (modelo IAA/PLANALSUCAR STOLF), segundo a metodologia de Stolf et al. (1983), realizando 60 testes em cada sistema até a profundidade de $60 \mathrm{~cm}$. Os cálculos foram realizados utilizando o programa computacional de Stolf (1991). Os valores obtidos em $\mathrm{Kgf} \mathrm{cm}^{-2}$ foram multiplicados pela constante 0,098 para a transformação em MPa.

A permeabilidade do solo à água foi determinada em permeâmetro de carga constante segundo modificações de Lima et al. (1990).

Os resultados obtidos dos atributos avaliados foram submetidos à análise de variância, sendo os efeitos de profundidade, sistemas de manejo e das interações sistemas x profundidade, avaliados pelo teste de Scott e Knott (SCOTT \& KNOTT, 1974) ao nível de probabilidade de $5 \%$.

\section{RESULTADOS E DISCUSSÃO}

\section{Estabilidade de agregados}

$\mathrm{Na}$ avaliação da estabilidade de agregados pelo DMG (Quadro 2), foi observado que de modo geral todos os sistemas de manejo estudados apresentaram valores superiores a $4 \mathrm{~mm}$. Os maiores valores de DMG ocorreram nas camadas de 0-10 e 10-20 cm, com médias de 4,70 e 4,75 $\mathrm{mm}$, respectivamente, apresentando redução na estabilidade de agregados expressa pelo DMG com o aumento da profundidade. Reduções nos valores de DMG em profundidade também foram observadas em trabalhos conduzidos por Beutler et al. (2001), D’Andréa (2001) e Silva \& Mielniczuk (1997), em sistemas de manejo. No entanto, mesmo havendo redução do diâmetro médio geométrico em profundidade, o valor médio observado na camada de $20-30 \mathrm{~cm}$ (4,58 mm) ainda apresenta uma elevada estabilidade de agregados nos diferentes sistemas estudados, sugerindo por meio desse atributo a manutenção da condição de qualidade de solo.

$\mathrm{Na}$ comparação dos valores de DMG entre os sistemas de manejo estudados e o $\mathrm{CN}$, não foram observadas diferenças significativas (Pd'0,05). Semelhança nos valores do DMG do solo sob sistemas de manejo que aplicam diferentes intensidades de revolvimento e sob cerrado nativo também foi observada por Figueiredo (1998). Entretanto, em trabalhos conduzidos por Alvarenga \& Davide (1999) e D’Andréa (2001) foram observadas reduções na estabilidade de agregados em sistemas revolvidos, em função das técnicas de manejo e principalmente do tempo de utilização, sendo observado pelo último autor a redução do DMG de 4,55 mm para 2,71 $\mathrm{mm}$ entre os sistemas cerrado nativo e o cultivo convencional de milho e soja com 15 anos de uso.

Nos sistemas com pouco tempo de implantação (CCB, CCBAM e CCM), e que praticam o revolvimento do solo, a provável explicação para a continuidade do equilíbrio com a condição natural, ou seja, a redução nãosignificativa desse atributo indicador da qualidade do solo, seria o pouco tempo de cultivo e o grande aporte de carbono orgânico (Quadro 1), oriundo do desmatamento do cerrado, o que provavelmente esta mantendo a estabilidade dos agregados nesses sistemas.

Já nos sistemas PDM e CE com longo tempo de cultivo, nos quais também não foi observada redução significativa desse atributo indicador da qualidade do solo, a provável explicação para a manutenção dessa condição esta relacionada ao não revolvimento do solo nos últimos anos e também ao acúmulo de carbono orgânico (palhada e liteira), (Quadro 1) nesses sistemas, além da capacidade agregante do sistema radicular da gramínea consorciada (Brachiaria brizanta) no sistema PDM. Observações de manutenção desta condição de equilíbrio entre o sistema de plantio direto e o cerrado nativo e a ação benéfica de gramíneas na estabilidade de agregados, também foram relatadas em Latossolos por Campos et al. (1995), Ros et al. (1997) e Silva et al. (1998), respectivamente.

Na concentração do tamanho dos agregados maior que $2 \mathrm{~mm}$ (Quadro 2), foi observado o mesmo comportamento estatístico verificado na análise do atributo DMG, não sendo observada diferença estatística significativa dos sistemas de manejo estudados em relação ao cerrado nativo, e sendo observada apenas diferença nas médias das profundidades analisadas, em que foi verificada redução na concentração de agregados com tamanho maior que $2 \mathrm{~mm}$ com o aumento da profundidade. Porém, foi observado que todos os sistemas apresentaram concentração de agregados maiores que $2 \mathrm{~mm}$ acima de $95 \%$, corroborando a alta estabilidade de agregados observada pelo atributo DMG.

\section{Densidade do solo}

Não foram verificadas diferenças estatísticas significativas nos valores de densidade do solo dos diferentes sistemas de manejo entre as profundidades analisadas. No entanto, diferença significativa (Pd'0,05) foi observada entre as médias das profundidades (Quadro 3).

Ciênc. agrotec., Lavras, v. 29, n. 4, p. 719-730, jul./ago., 2005 
QUADRO 2 - Estabilidade de agregados determinada pelo diâmetro médio geométrico (DMG) e agregados maiores que 2 mm em um Latossolo Vermelho-Amarelo Ácrico típico sob diferentes sistemas de manejo em relação ao cerrado nativo.

\begin{tabular}{|c|c|c|c|c|c|c|c|}
\hline \multirow{2}{*}{ Profundidade $(\mathrm{cm})$} & \multicolumn{6}{|c|}{ Sistema de manejo ${ }^{(1)}$} & \multirow{2}{*}{ Média } \\
\hline & CCB & CCBAM & CCM & PDM & $\mathbf{C E}$ & $\mathbf{C N}$ & \\
\hline & \multicolumn{6}{|c|}{ 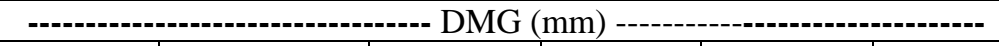 } & \\
\hline $0-10$ & 4,54 & 4,62 & 4,69 & 4,76 & 4,73 & 4,87 & $4,70 \mathrm{a}$ \\
\hline $10-20$ & 4,67 & 4,85 & 4,68 & 4,64 & 4,77 & 4,87 & $4,75 \mathrm{a}$ \\
\hline $20-30$ & 4,55 & 4,66 & 4,36 & 4,67 & 4,57 & 4,68 & $4,58 \mathrm{~b}$ \\
\hline Média & 4,59 & 4,71 & 4,58 & 4,69 & 4,69 & 4,81 & \\
\hline C.V. Sistema & $(\%)$ & 3,58 & & & & & \\
\hline \multirow[t]{2}{*}{ C.V. Total } & $(\%)$ & 2,47 & & & & & \\
\hline & \multicolumn{6}{|c|}{ 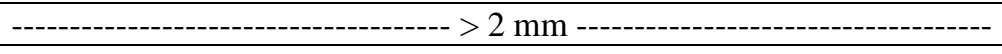 } & \\
\hline $0-10$ & 96,8 & 96,9 & 97,4 & 98,4 & 98,2 & 99,4 & $97,7 \mathrm{a}$ \\
\hline $10-20$ & 97,7 & 98,9 & 97,6 & 97,2 & 98,3 & 99,3 & $98,2 \mathrm{a}$ \\
\hline $20-30$ & 95,9 & 97,2 & 98,0 & 97,3 & 96,6 & 97,4 & $96,7 b$ \\
\hline Média & 96,8 & 97,7 & 97,7 & 97,6 & 97,7 & 98,7 & \\
\hline C.V. Sistema & $(\%)$ & 1,6 & & & & & \\
\hline C.V. Total & $(\%)$ & 1,1 & & & & & \\
\hline
\end{tabular}

(1) CCB (Cultivo convencional de batata); CCBAM (Cultivo convencional de batata, sucedido por aveia após uma subsolagem, em rotação com milho); CCM: (Cultivo convencional com milho em rotação com feijão); PDM (Plantio direto com milho, após cultivos convencionais com batata, arroz e milho); CE (Cultivo de eucalipto) e CN (Cerrado nativo). Médias seguidas pela mesma letra na coluna, não diferem estatisticamente entre si, pelo teste de Scott e Knott ao nível de $5 \%$ de probabilidade.

QUADRO 3 - Densidade em um Latossolo Vermelho-Amarelo Ácrico típico sob diferentes sistemas de manejo em relação ao cerrado nativo.

\begin{tabular}{|c|c|c|c|c|c|c|c|}
\hline \multirow{2}{*}{ Profundidade $(\mathrm{cm})$} & \multicolumn{6}{|c|}{ Sistema de manejo ${ }^{(1)}$} & \multirow{2}{*}{ Média } \\
\hline & CCB & CCBAM & $\mathbf{C C M}$ & PDM & $\mathbf{C E}$ & $\mathbf{C N}$ & \\
\hline & \multicolumn{6}{|c|}{ 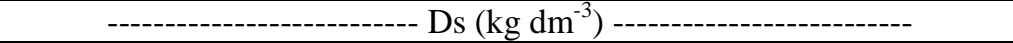 } & \\
\hline $0-10$ & 1,03 & 1,17 & 1,08 & 1,17 & 1,04 & 1,08 & $1,09 \mathrm{~b}$ \\
\hline $10-20$ & 1,17 & 1,22 & 1,05 & 1,18 & 1,15 & 1,11 & $1,15 \mathrm{a}$ \\
\hline $20-30$ & 1,11 & 1,13 & 1,03 & 1,14 & 1,02 & 1,05 & $1,08 \mathrm{~b}$ \\
\hline Média & $1,10 \mathrm{~B}$ & $1,17 \mathrm{~A}$ & $1,05 \mathrm{~B}$ & $1,16 \mathrm{~A}$ & $1,07 \mathrm{~B}$ & $1,08 \mathrm{~B}$ & \\
\hline C.V. Sistema & $(\%)$ & 6,3 & & & & & \\
\hline C.V. Total & $(\%)$ & 4,7 & & & & & \\
\hline
\end{tabular}

(1) CCB (Cultivo convencional de batata); CCBAM (Cultivo convencional de batata, sucedido por aveia após uma subsolagem, em rotação com milho); CCM: (Cultivo convencional com milho em rotação com feijão); PDM (Plantio direto com milho, após cultivos convencionais com batata, arroz e milho); CE (Cultivo de eucalipto) e CN (Cerrado nativo). Médias seguidas pela mesma letra, minúscula na coluna e maiúscula na linha, não diferem estatisticamente entre si, pelo teste de Scott e Knott ao nível de 5\% de probabilidade. 
$\mathrm{Na}$ camada de 10-20 $\mathrm{cm}$ foram observados os maiores valores de densidade do solo. No entanto, esse aumento apresentou-se generalizado, não podendo ser considerado como uma alteração em função unicamente do sistema de manejo. Já os menores valores da densidade do solo foram observados nas camadas de 0-10 e 20-30 cm. Na camada de 0-10 cm essa redução nos valores desse atributo indicadores da qualidade do solo, pode ser explicada pelo maior revolvimento do solo durante o preparo nos sistemas convencionais (CCB, CCBAM e CCM) e por uma maior estruturação do solo pela ação dos microrganismos decompositores da matéria orgânica nos sistemas não revolvidos (PDM, CE e CN), além da ação dos sistemas radiculares ao longo do tempo de exploração.

Quanto a semelhança observada nos valores da densidade do solo na camada de $20-30 \mathrm{~cm}$, sugere que os sistemas convencionais com pouco tempo de implantação (CCB, CCBAM e CCM) não alteraram ainda a estrutura do solo o suficiente para diferir da condição de equilíbrio, o $\mathrm{CN}$, e que os sistemas não revolvidos com longo tempo de implantação (PDM e CE) poderiam estar possivelmente restabelecendo a condição de equilíbrio com o cerrado nativo.

Na comparação dos diferentes sistemas de manejo estudados com o $\mathrm{CN}$, foi observada diferença significativa (Pd'0,05) apenas nas médias dos sistemas CCBAM e PDM. Nesses sistemas foram observados valores de densidade do solo estatisticamente superiores ao $\mathrm{CN}$. No sistema PDM, o aumento da densidade do solo pode ser explicado tanto pela acomodação das partículas do solo após a sua implantação como pelo o tráfego de máquinas pesadas nas operações de plantio e colheita (SILVA et al., 2000; TORMENA et al., 1998). Já no sistema CCBAM, esse comportamento pode ser explicado pela desagregação intensiva com um sucessivo empacotamento das partículas do solo, decorrente de um intenso uso de máquinas e implementos agrícolas, que em muitas vezes se encontram sob condições inadequadas de umidade do solo. Essa prática, segundo Dias Júnior \& Pierce (1996), leva à degradação estrutural do solo e, conseqüentemente, ao aumento de sua densidade. Entretanto, mesmo sendo observada semelhança estatística nesses sistemas, ressalta-se que, por se tratar de um tempo de uso maior no sistema PDM (15 anos), infere-se um maior potencial de redução da qualidade do solo no sistema CCBAM (2 anos de uso). Além disso, algumas características do PDM como a formação da palhada e o aumento do teor de carbono no solo (Quadro 1), elevam a capacidade de suporte de carga do solo, aumentando a resistência à elevação da densidade do solo nesse sistema.

\section{Porosidade total e distribuição de poros por tamanho}

Não foram verificadas diferenças estatísticas significativas nos valores de volume total de poros (VTP) dos diferentes sistemas de manejo entre as profundidades analisadas. No entanto, diferença significativa (Pd'0,05) foi observada entre as médias das profundidades (Quadro 4), sendo maiores na profundidade de $20-30 \mathrm{~cm}$ e menores na de 10-20 cm. Os baixos valores de VTP observados na camada de 10-20 cm são decorrentes do aumento na densidade do solo nessa mesma profundidade (Quadro 3), o que assim como o comportamento da densidade do solo também não deve ser considerado como de efeito unicamente do sistema de manejo, por apresentar-se também no sistema CN.

Reduções no volume total de poros na camada de 10-20 cm também foram observadas em solos da mesma região por Chagas et al. (1997), e em outras regiões de cerrado por Beutler et al. (2001) e D’Andréa (2001). Assim, nesse tipo de estudo recomenda-se o uso de um sistema em equilíbrio como padrão comparativo, uma vez que, na ausência do mesmo, este efeito ficaria despercebido ou inserido na densidade do solo como efeito unicamente do manejo, sendo erroneamente discutido como redução da qualidade do solo.

Os menores valores de volume total de poros (VTP) foram observados nos sistemas CCB, CCBAM e PDM, os quais diferiram estatisticamente (Pd'0,05) do sistema $\mathrm{CN}$. $\mathrm{O}$ CCB é um sistema exigente em preparo, o que leva à pulverização do solo durante os tratos culturais e colheita. Essa mobilização do solo favorece o arranjamento das partículas do solo tanto natural inicialmente, quanto por tráfego de máquinas na realização dos tratos culturais, como observado por Stone \& Silveira (2001), em outros sistemas convencionais de manejo intensamente revolvidos.

No sistema CCBAM, no qual cultivou-se batata em sistema convencional no primeiro ano, aveia em sucessão e milho em rotação no segundo ano, e portanto, podendo ser considerado como uma evolução conservacionista do sistema CCB, que praticou apenas o cultivo convencional de batata por um ano, não foi observada melhoria na qualidade do solo avaliada através do atributo indicador volume total de poros em relação ao sistema CCB. Entretanto, essa semelhança com o sistema CCB, não deve invalidar o uso de práticas conservacionista de manejo

Ciênc. agrotec., Lavras, v. 29, n. 4, p. 719-730, jul./ago., 2005 
QUADRO 4 - Volume total de poros e microporosidade em um Latossolo Vermelho-Amarelo Ácrico típico sob diferentes sistemas de manejo em relação ao cerrado nativo.

\begin{tabular}{|c|c|c|c|c|c|c|c|}
\hline \multirow{2}{*}{$\begin{array}{l}\text { Profundidades } \\
\text { (cm) }\end{array}$} & \multicolumn{6}{|c|}{ Sistema de manejo $^{(1)}$} & \multirow{2}{*}{ Média } \\
\hline & CCB & CСВАМ & CCM & PDM & $\mathbf{C E}$ & $\mathbf{C N}$ & \\
\hline & \multicolumn{6}{|c|}{ - } & \\
\hline $0-10$ & 57 & 53 & 55 & 52 & 58 & 56 & $55,2 \mathrm{~b}$ \\
\hline $10-20$ & 52 & 51 & 56 & 52 & 53 & 55 & $53,2 \mathrm{c}$ \\
\hline $20-30$ & 56 & 55 & 57 & 55 & 59 & 58 & $57,7 \mathrm{a}$ \\
\hline Média & $55,0 \mathrm{~B}$ & $53,0 \mathrm{~B}$ & $56,0 \mathrm{~A}$ & $53,8 \mathrm{~B}$ & $56,7 \mathrm{~A}$ & $56,7 \mathrm{~A}$ & \\
\hline C.V. Sistema & $(\%)$ & 5,4 & & & & & \\
\hline \multirow[t]{2}{*}{ C.V. Total } & $(\%)$ & 3,6 & & & & & \\
\hline & \multicolumn{6}{|c|}{ - } & \\
\hline $0-10$ & $38 \mathrm{bB}$ & $43 \mathrm{aA}$ & $44 \mathrm{aA}$ & $47 \mathrm{aA}$ & $40 \mathrm{bB}$ & $38 \mathrm{aB}$ & 41 \\
\hline $10-20$ & $43 \mathrm{aA}$ & $41 \mathrm{aB}$ & $46 \mathrm{aA}$ & $47 \mathrm{aA}$ & $46 \mathrm{aA}$ & $37 \mathrm{aC}$ & 43 \\
\hline $20-30$ & $44 \mathrm{aA}$ & $43 \mathrm{aA}$ & $46 \mathrm{aA}$ & $46 \mathrm{aA}$ & $47 \mathrm{aA}$ & $38 \mathrm{aB}$ & 44 \\
\hline Média & 41 & 42 & 45 & 46 & 44 & 37 & \\
\hline C.V. Sistema & $(\%)$ & 7,4 & & & & & \\
\hline C.V. Total & $(\%)$ & 5,2 & & & & & \\
\hline
\end{tabular}

(1) CCB (Cultivo convencional de batata); CCBAM (Cultivo convencional de batata sucedido por aveia após uma subsolagem, em rotação com milho); CCM: (Cultivo convencional com milho em rotação com feijão); PDM (Plantio direto com milho, após cultivos convencionais com batata, arroz e milho); CE (Cultivo de eucalipto) e CN (Cerrado nativo). Médias seguidas pela mesma letra, minúscula na coluna e maiúscula na linha, não diferem estatisticamente entre si, pelo teste de Scott e Knott ao nível de 5\% de probabilidade.

como a sucessão e a rotação de cultivo. Com esse manejo conservacionista, pode estar ocorrendo uma redução na velocidade de acomodação das partículas do solo, mediante o efeito estruturador do sistema radicular das gramíneas, no caso a aveia (ALBUQUERQUE et al., 1995; SILVA \& ROSOLEM, 2001; STONE \& SILVEIRA, 2001; TORMENA et al., 1998). O efeito benéfico das gramíneas para a formação de agregados com conseqüente aumento da porosidade total do solo também se aplica no sistema PDM, o qual, além do milho como cultura principal, realizou-se a sobre semeadura com capim Brachiaria brizantha nos dois últimos anos de cultivo do milho. Observações de efeitos benéficos dessa gramínea na estruturação do solo também foram verificadas por Silva \& Mielniczuck (1997).

Analisando o efeito de sistemas dentro de profundidade, foi observado na camada de $0-10 \mathrm{~cm}$ os maiores valores de microporosidade nos sistemas CCBAM, CCM e PDM, (43, 44 e 47\%, respectivamente) em relação ao $\mathrm{CN}$, no qual foi observado apenas $38 \%$ de microporosidade. Isso, está refletindo a interferência do sistema de manejo na redução do tamanho dos poros, uma vez que não foi observada diferença significativa nos valores de VTP entre esses respectivos sistemas e o $\mathrm{CN}$ na mesma profundidade (Quadro 4). Já no sistema CCB, no qual não foi observada diferença significativa maior em relação ao $\mathrm{CN}$, mas que também não diferiu estatisticamente nos valores de VTP em relação ao $\mathrm{CN}$, a explicação é dada em função de um intenso revolvimento do solo durante a colheita que antecedeu o período de amostragem do solo.

Na camada de 10-20 cm, a observação foi generalizada e todos os sistemas apresentaram valores de microporosidade superiores ao observado no $\mathrm{CN}$. No entanto, também não foi observada diferença estatística significativa nos valores de VTP em relação ao CN, permitindo a inferência de que esteja ocorrendo apenas uma alteração na distribuição de poros por tamanho, em 
que a macroporosidade está sendo reduzida em relação ao CN. No sistema CCBAM, esse efeito foi menos expressivo. Nesse sistema, foi observada uma redução estatística nãosignificativa no valor de VTP (Quadro 4), que associada ao cultivo em sucessão de aveia e a rotação com o cultivo de milho, provavelmente estão promovendo menores acréscimos nos valores da microporosidade. Também na camada de 20-30 cm todos os sistemas apresentaram diferença estatística significativa maior em relação ao $\mathrm{CN}$, indicando também que todos os sistemas tendem a diminuir a macroporosidade em relação ao sistema natural $(\mathrm{CN})$.

Analisando o efeito das profundidades dentro de cada sistema de manejo, foram observadas apenas uma redução nos valores da microporosidade nos sistemas CCB e CE na camada de 0-10 cm. Esse efeito no sistema CCB, pode ser decorrente do revolvimento do solo no momento da colheita, realizada em período próximo as amostragens do solo nesse sistema. No sistema CE, a provável explicação dessa menor microporosidade em relação as camadas inferiores, pode estar relacionada com um aumento na macroporosidade, uma vez que não foi observada diferença estatística nos valores da microporosidade no efeito de sistemas dentro de profundidade em relação ao $\mathrm{CN}$. Nesse sistema CE, o não revolvimento do solo ao longo de 13 anos após a implantação da cultura de eucalipto, levou a um acúmulo nessa camada $0-10 \mathrm{~cm}$ de carbono orgânico (Quadro 1), e raízes, porém, não quantificadas, que estão promovendo uma maior agregação do solo (Quadro 2), e que provavelmente estão refletindo no aumento da macroporosidade. Nos sistemas CCBAM, CCM, PDM e $\mathrm{CN}$, não foram verificadas diferenças significativas.

\section{Resistência do solo à penetração vertical}

Foi verificado um aumento da resistência à penetração com o aumento da profundidade (Figura 1). Na camada de 0-10 cm, os maiores valores de resistência à penetração foram observados nos sistemas CE e PDM (1,6 e 1,5 $\mathrm{MPa}$, respectivamente). No entanto, mesmo apresentando valores mais elevados nessa camada, estes sistemas podem ainda não oferecer resistência significativa ao desenvolvimento radicular com efeito restritivo para o aumento da produtividade. Segundo Ehlers et al. (1983), em sistemas conservacionistas, que apresentam menor revolvimento no solo e que acumulam mais matéria orgânica, existe uma tendência em haver maior eficiência de raízes e microrganismos na sua ação estruturadora do solo, permitindo maior amplitude dos limites de resistência à penetração, podendo ser considerado nessas condições o limite restritivo de $5 \mathrm{MPa}$.

Na camada de 10-20 cm, os sistemas CEe PDM também apresentaram os maiores valores de resistência à penetração, comparativamente aos demais sistemas de manejo estudados. No entanto, nessa profundidade todos os sistemas de manejo estudados conferiram resistência do solo à penetração inferior ao CN (3,0 MPa). Entretanto, segundo Ehlers et al. (1983), estes sistemas ainda não oferecem restrição significativa ao desenvolvimento radicular.

No sistema PDM, foi observado um maior acréscimo da resistência à penetração na camada de $10-20 \mathrm{~cm}$, variando de 1,6 MPa a 2,7 MPa dos $10 \mathrm{~cm}$ aos $20 \mathrm{~cm}$ de profundidade, respectivamente. A explicação para esse comportamento baseia-se no histórico de uso pouco conservacionista durante o período sob sistema convencional, que se iniciou com cultivo de batata, (safra 86/87), seguido por arroz (87/ 89) e posteriormente, um monocultivo com milho até a safra 96, período em que foi implantado o sistema PDM.

Mediante esse histórico, mesmo não sendo realizados testes de resistência à penetração anteriormente, nos leva a sugerir que esse acréscimo, em relação ao $\mathrm{CN}$, não se deve unicamente ao sistema PDM, mas também a um efeito acumulado do período sob cultivo convencional, em que depois de cessada a mobilização do solo com preparo convencional, ocorreu um arranjamento maior das partículas do solo reduzindo os valores do volume total de poros (Quadro 4) e aumentando os valores da densidade (Quadro 3), com o conseqüente aumento da resistência do solo à penetração. Corrêa (1985), Vieira (1981) e Vieira \& Muzilli (1995) também relatam sobre o arranjamento natural do solo, após a adoção do sistema de plantio direto, quando não é mais mobilizado sob sistema convencional.

$\mathrm{Na}$ camada de 20-30 cm, foi observado o mesmo comportamento da camada de $10-20 \mathrm{~cm}$, ocorrendo apenas acréscimos nos valores da resistência à penetração em relação a camada anterior em todos os sistemas, sendo mais expressivos nos sistemas CE e PDM. Segundo Arshad et al. (1996) e Grant \& Lanfond (1993), que relatam 1,5 e 2,0 $\mathrm{MPa}$, respectivamente, como valores críticos restritivos aos desenvolvimento radicular em sistemas convencionais, os valores apresentados nestes sistemas, conferem uma camada impeditiva ao desenvolvimento radicular, ao contrário de quando referido por Ehlers et al. (1983), por serem sistemas conservacionistas.

Nas profundidades maiores que $30 \mathrm{~cm}$, consideradas como de menores transformações em relação às camadas superficiais, todos os sistemas estudados exceto o sistema em equilíbrio (CN) apresentaram

Ciênc. agrotec., Lavras, v. 29, n. 4, p. 719-730, jul./ago., 2005 
Resistência à Penetração (MPa)

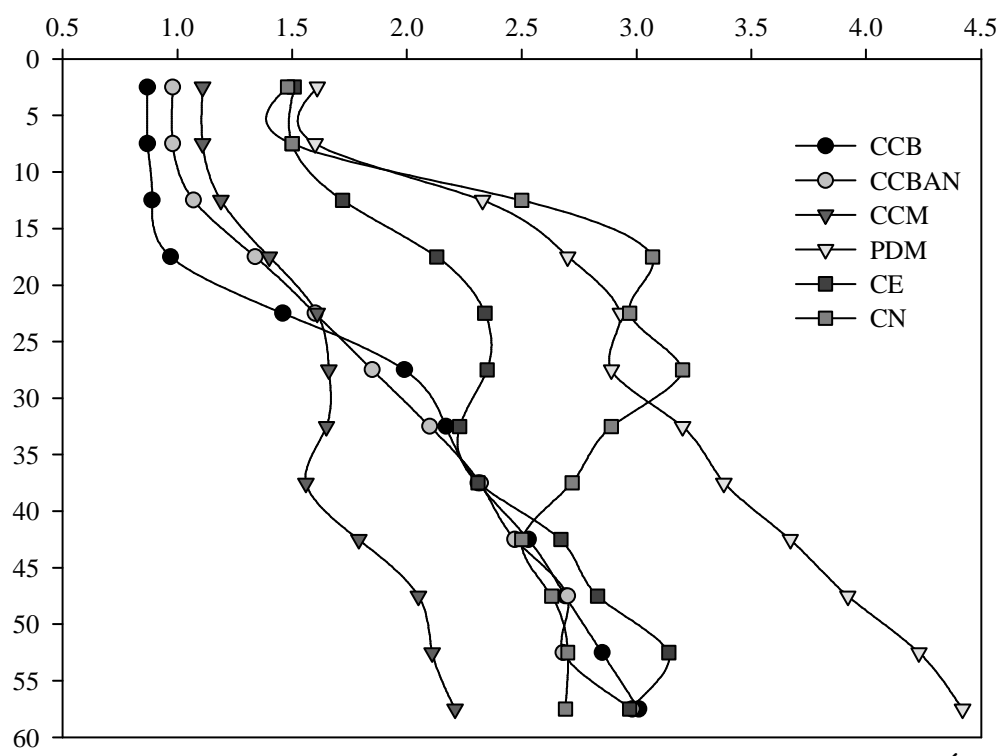

FIGURA 1 - Resistência do solo à penetração em um Latossolo Vermelho-Amarelo Ácrico típico sob diferentes sistemas de manejo (CCB: Cultivo convencional de batata CCBAM: Cultivo convencional de batata, sucedido por aveia após uma subsolagem, em rotação com milho; CCM: Cultivo convencional com milho em rotação com feijão; PDM: Plantio direto com milho, após os cultivos convencionais de batata, arroz e milho e CE: Cultivo de eucalipto) em relação ao $\mathrm{CN}$ : cerrado nativo.

acréscimos da resistência à penetração com o aumento da profundidade. Os maiores acréscimos foram observados nos sistemas CCB, CCBAM e PDM, variando os valores de resistência à penetração de 1,6, 1,8 e 2,8 MPa para 2,1, 2,9 e 4,4 $\mathrm{MPa}$, respectivamente. Ressalta-se que, coincidentemente, esses sistemas cultivaram batata em seu primeiro ciclo de cultivo, o que provavelmente está relacionado com a semelhança de comportamento entre esses sistemas, caraterizando assim o alto efeito degradativo na qualidade do solo pelo manejo aplicado para o cultivo de batata.

\section{Permeabilidade do solo à água}

$\mathrm{Na}$ Figura 2, observam-se os valores de permeabilidade do solo á água no sistema em equilíbrio (CN) e nos sistemas de manejo estudados. O cerrado nativo destacou-se com valor bastante elevado de permeabilidade em relação aos sistemas de manejados, em consonância com o comportamento dos teores de carbono orgânico total (Quadro 1) e demais atributos físicos (Quadros 2, 3 e 4, Figura 1).

Não foram observadas diferenças significativas na permeabilidade do solo à água entre os sistemas CCB, CCBM, CCM e PDM (Figura 2), sendo os valores enquadrados na classe lenta a moderada, em relação aos sistemas CE e CN as classificações foram moderada e moderada a rápida, respectivamente. Resultados semelhantes foram observados por Beuther et al. (2001).

Os valores de permeabilidade do solo á água observados na Figura 2 indicam uma redução significativa, em média $75 \%$, quando comparados com o sistema em equilíbrio $(\mathrm{CN})$. As substituições da vegetação nativas por lavouras, com a adoção de manejo inadequado, têm propiciado alterações significativas neste atributo, modificando todo um ciclo da água na bacia hidrográfica. Entretanto, com a adoção de sistemas de manejo conservacionistas (CCBAM e PDM) existe uma tendência do quadro inverter.

Os atributos físicos estudados apresentaram boa performance como indicadores da qualidade do solo, distingüindo os efeitos proporcionados pelos sistemas de manejo em relação ao sistema em equilíbrio, contribuindo para o monitoramento do manejo sustentável de solos da região. 


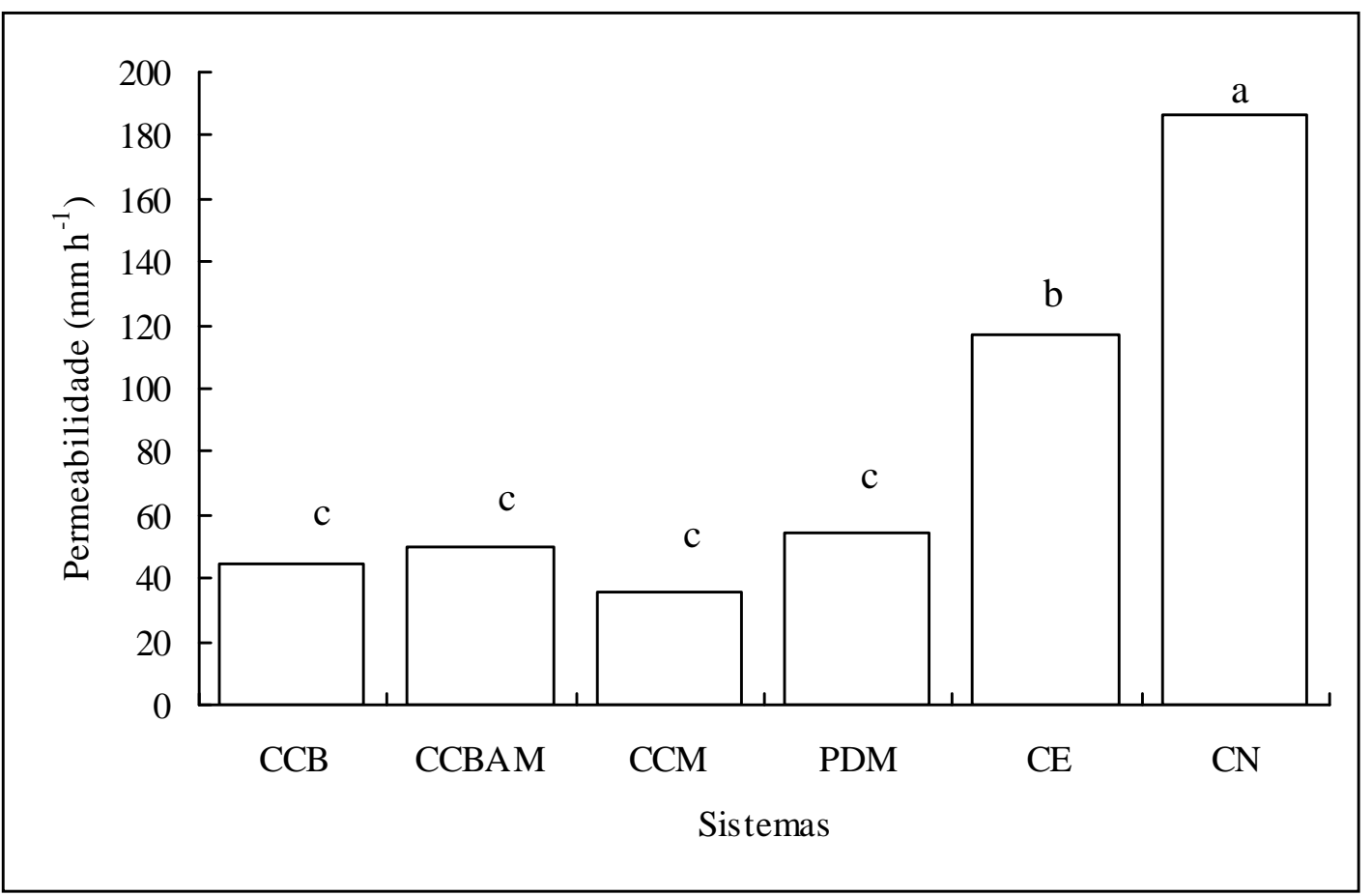

FIGURA 2 - Permeabilidade do solo à água em um Latossolo Vermelho-Amarelo Ácrico típico em diferentes sistemas de manejo. (CCB: Cultivo convencional de batata; CCBAM: Cultivo convencional de batata sucedido por aveia após uma subsolagem, e rotacionado com milho; CCM: Cultivo convencional com milho em rotação com feijão; PDM: Plantio direto com milho, após os cultivos convencionais com batata, arroz e milho; CE: Cultivo de eucalipto e CN: Cerrado nativo. Médias seguidas pela mesma letra não diferem estatisticamente entre si, pelo teste de Scott e Knott ao nível de $5 \%$ de probabilidade).

\section{CONCLUSÕES}

Os sistemas convencionais CCM, CCB e CCBAM com pouco tempo de implantação não alteraram a estabilidade de agregados o suficiente para diferir do cerrado nativo, enquanto os sistemas PDM e CE com menor revolvimento estão estabelecendo uma nova condição de equilíbrio.

O menor revolvimento do solo, o acúmulo de carbono orgânico e a ação agregante do sistema radicular das gramíneas foram benéficos na manutenção da qualidade do solo.

A resistência do solo à penetração foi reduzida nas camadas superficiais nos sistemas convencionais em função da mobilização do solo, e os sistemas que coincidentemente tiveram cultivo de batata inicialmente, apresentaram as maiores resistências à penetração com o aumento da profundidade.
Os sistemas manejados apresentaram valores de permeabilidade do solo á água bem inferiores àqueles observados sob cerrado nativo.

\section{REFERÊNCIAS BIBLIOGRÁFICAS}

ALBUQUERQUE, J. A.; REINERT, D. J.; FIORIN, J. E.; RUEDELL, J.; PETRERE, C.; FONTINELLI, F. Rotação de culturas e sistemas de manejo do solo: efeito sobre a forma da estrutura do solo ao final de sete anos. Revista Brasileira de Ciência do Solo, Campinas, v. 19, n. 1, p. 115119, jan./abr. 1995.

ALVARENGA, M. I. N.; DAVIDE,A.C. Características físicas e químicas de um Latossolo Vermelho-Escuros e a sustentabilidade de agroecossistemas. Revista Brasileira de Ciência do Solo, Viçosa, v. 23, n. 4, p. 933-942, out./dez. 1999. 
ANJOS, J. T.; UBERTI, A. A. A.; VIZZOTO, V. J.; LEITE, G. B.; KRIEGER, M. Propriedades físicas em solos sob diferentes sistemas de uso e manejo. Revista Brasileira de Ciências de Solo, Campinas, v. 18, n. 0, p. 139-145, 1994.

ARSHAD, M. A.; LOWERY, B.; GROSSMAN, R. Physical test for monitoring soil quality. In: DORAN, J. W.; JONES, A. J. (Eds.). Methods for assessing soil quality. Madison: SSSA, 1996. (Special Publication Number, 49).

BEUTLER, A. N.; SILVA, M. L. N.; CURI, N.; FERREIRA, M. M.; PEREIRA FILHO, I. A.; CRUZ, J. C. Resistência à penetração e permeabilidade de Latossolo Vermelho Distrófico típico sob sistemas de manejo na região dos cerrados. Revista Brasileira de Ciência do Solo, Viçosa, v. 25, n. 1, p. 167-177, jan./mar. 2001.

BLAKE, G. R.; HARTGE, K. H. Bulk density. In: KLUTE, A. (Ed.). Methods of soil analysis. 2. ed. Madison: American Society of Agronomy, 1986. v. 1, p. 363-375.

BRASIL. Ministério das Minas e Energia. Projeto Radambrasil. Levantamento dos recursos naturais: folhas SF 23/24. Rio de Janeiro, 1983. v. 32, 775 p.

CAMPOS, B.C.; REINERT, D. J.; NICOLODI, R.; RUEDELL, J.; PETRERE, C. Estabilidade estrutural de um Latossolo Vermelho Escuro distrófico após sete anos de rotação de culturas e sistemas de manejo. Revista Brasileira de Ciência do solo, Campinas, v. 19, n. 1, p. 121-126, jan./abr. 1995.

CORRÊA, J. C. Efeito de métodos de métodos de cultivo em algumas propriedades físicas de um Latossolo Amarelo muito argiloso do estado do Amazonas. Pesquisa Agropecuária Brasileira, Brasília, v. 20, p. 1317-1322, 1985.

CHAGAS, C. S.; CURI, N.; DUARTE, M. N.; MOTTA, P. E. F.; LIMA, J. M. Orientação das camadas de rocha metapelíticas pobres na gênese de latossolos sob cerrado. Pesquisa Agropecuária Brasileira, Brasília, v. 32, n. 5, p. 539-548, maio 1997.

D'ANDRÉA, A. F. Atributos indicadores da qualidade do solo em sistemas de manejo no Sul de Goiás. 2001. 104 p. Dissertação (Mestrado em Solos e Nutrição de Plantas) Universidade Federal de Lavras, Lavras, 2001.
DANIELSON, R. E.; SUTHERLAND, P. L. Porosity. In: KLUTE, A. (Ed.). Methods of soil analysis: physical and mineralogical methods. Madison: ASA, 1986. p. 443-461.

DIAS JÚNIOR, M. S.; PIERCE, F. J. O processo de compactação do solo e sua modelagem. Revista Brasileira de Ciência de Solo, Campinas, v. 20, n. 2, p. 175-182, maio/ ago. 1996.

DORAN, J. W.; PARKIN, T. B. Defining and assessing soil quality. In: DORAN, J. W.; COLEMAN, D. C.; BEZDICEK, D. F.; STEWART, B. A. Defining soil quality for a sustainable environment. Madison: Soil Science Society of America, 1994. p. 3-21. (SSSA Special publication, 35).

EHLERS, W.; KOPKE, V.; HESSE, F; BOHM, W. Pnetration resistance an root growth of aots in tilled and untilled loess soil. Soil Tillage Research, Amsterdam, v. 3, p. 261-275, 1983.

EMPRESA BRASILEIRA DE PESQUISA AGROPECUÁRIA. Serviço Nacional de Levantamento e Conservação de Solo. Manual de métodos de análise de solo. Rio de Janeiro: Ministério da Agricultura, 1997. 212 p.

EMPRESA BRASILEIRA DE PESQUISA AGROPECUÁRIA. Centro Nacional de Pesquisa em Solo. Sistema brasileiro de classificação de solo. Brasília; Rio de Janeiro, 1999. 412 p.

FIGUEIREDO, L. H. A. Propriedades físicas e mecânicas de um LR. submetido a diferentes sistemas de manejo. 1998. Dissertação (Mestrado em Solos e Nutrição de Plantas) - Universidade Federal de Lavras, Lavras, 1998.

GRANT, C. A.; LANFOND, G. P. The effects of tillage systems and crop sequences on soil bulk density and penetration resistence on a clay soil in southern Saskatchewan. Canadian Journal of Soil Science, Ottawa, v. 73, n. 2, p. 223-232, May 1993.

LIMA, J. M.; CURI, N.; RESENDE, M.; SANTANA, D. P. Dispersão do material de solo em água para avaliação indireta da erodibilidade de latossolos. Revista Brasileira de Ciência do Solo, Campinas, v. 14, n. 1, p. 85-90, jan./abr. 1990. 
RACHWAL, M. F. G; DEDECEK, R.A. Influência da aeração e da disponibilidade hídrica em Cambissolos e Latossolos com diferentes níveis de erosão sobre a produtividade e a qualidade da cultura da batata. Revista Brasileira de Ciência do Solo, Campinas, v. 20, n. 3, p. 485-491, set./dez. 1996.

ROS, C. O. da; SECCO, D.; FIORIN, J. E.; PETRERE, C.; CADORE, M. A.; PASA, L. Manejo do solo a partir de campo nativo: efeito sobre a forma e estabilidade da estrutura ao final de cinco anos. Revista Brasileira de Ciência do Solo, Campinas, v. 21, n. 2, p. 241-247, abr./jun. 1997.

SCOTT, A. J.; KNOTT, M. Accouter analysis methods for grouping means in the analysis of variants. Biometrics, Washington, v. 30, n. 3, p. 507-512, Sept. 1974.

SEYBOLD, C. A.; HERRICK, J. E.; BREJDA, J. J. Soil resilienc: a fundamental component of soil quality. Soil Science, Baltimore, v. 164, p. 224-234, 1999.

SILVA, I. F.; MIELNICZUK, J. Ação do sistema radicular de plantas na formação e estabilização de agregados do solo. Revista Brasileira de Ciência do Solo, Campinas, v. 21, n. 1, p. 113-117, jan./mar. 1997.

SILVA, M.L. N.; BLANCANEAUX, P. H.; CURI, N.; LIMA, J. M. de; MARQUES, J. J. G. de S. e M.; CARVALHO, A. M. de. Estabilidade e resistência de agregados de Latossolo Vermelho-Escuro cultivado com sucessão milho-adubo verde. Pesquisa Agropecuária Brasileira, Brasília, v. 33, n. 1, p. 97-103, jan. 1998.

SILVA, V. R.; REINERT, D. J.; REICHERT, J. M. Densidade do solo, atributos químicos e sistema radicular do milho afetados pelo pastejo e manejo do solo. Revista Brasileira de Ciência do Solo, Viçosa, v. 24, n. 1, p. 191-199, jan./mar. 2000.

SILVA, R. H.; ROSOLEM, C. A. Crescimento radicular de espécies utilizadas como cobertura decorrente da compactação do solo. Revista Brasileira de Ciência do Solo, Viçosa, v. 25, n. 2, p. 253-260, maio/jun. 2001.

STOLF, R. Teoria e teste experimental de fórmulas de transformação dos dados de penetrômetro de impacto em resistência do solo. Revista Brasileira de Ciência do solo, Campinas, v. 15, n. 3, p. 229-235, set./dez. 1991.

STOLF, R.; FERNANDES, J.; FURLANI NETO, V. L. Recomendação para uso do penetrômetro de impacto, modelo IAA/Planalsucar - Stolf. São Paulo: MIC/IAA/ PNMCA-Planalsucar, 1983. 8 p. (Série penetrômetro de impacto-Boletim, 1).

STONE, L. F.; SILVEIRA, P. M. Efeitos do sistema de preparo e da rotação de culturas na porosidade e densidade do solo. Revista Brasileira de Ciências do Solo, Viçosa, v. 25, n. 2, p. 395-401, abr.jun. 2001.

TORMENA, C. A.; ROLOFF, G.; SÁ, J. C. M. Propriedades físicas do solo sob plantio direto influenciadas por calagem, preparo inicial e tráfego. Revista Brasileira de Ciências do Solo, Viçosa, v. 22, n. 2, p. 301-309, abr.jun. 1998.

VIEIRA, M. J. Propriedades físicas do solo. In: INSTITUTO AGRONÔMICO DO PARANÁ. Plantio direto no Estado do Paraná. Londrina, 1981. p. 19-32. (IAPAR. Circular, 23).

VIEIRA, M. J.; MUZILLI, O. Características físicas de um Latossolo Vermelho-Escuro sob diferentes sistemas de manejo. Pesquisa Agropecuária Brasileira, Brasília, v. 19, n. 7, p. 873-882, jul. 1995. 